\title{
THE DENSITY FUNCTION OF THE SOLUTION OF A TWO-POINT BOUNDARY VALUE PROBLEM CONTAINING SMALL STOCHASTIC PROCESSES*
}

\author{
BY \\ NING-MAO XIA ${ }^{1}$ \\ East China Institute of Chemical Technology, Shanghai
}

Abstract. This paper concerns a two-point boundary value problem for an $m$ thorder system of ordinary differential equations containing a vector stochastic process

$$
\boldsymbol{\xi}(t, \omega)=\boldsymbol{\xi}_{0}(t)+\varepsilon \boldsymbol{\xi}_{1}(t, \omega)+\varepsilon^{2} \boldsymbol{\xi}_{2}(t, \omega)+\cdots .
$$

When $\varepsilon$ is small, the existence and the asymptotic properties of the solution can be obtained by means of the shooting method, and its density function can be determined by solving a sequence of first-order deterministic partial differential equations.

1. Introduction. Random differential equations are of great importance both for theoretical reasons and because of their practical applications. For random initial value problems many important and interesting facts have been discovered; see [1, $3,4,5,8]$, for example. However, for random boundary value problems only a relatively few results are known $[2,6,9]$.

In this paper we will consider the problem

$$
\begin{gathered}
\frac{d \mathbf{x}(t)}{d t}=\mathbf{f}(t, \mathbf{x}(t), \boldsymbol{\xi}(t, \omega)), \\
\mathbf{A x}(0)+\mathbf{B x}(1)=\alpha(\omega),
\end{gathered}
$$

where $\alpha(\omega)$ and $\boldsymbol{\xi}(t, \omega)$ are $m \times 1$ random vectors defined on an underlying probability space $(\Omega, \mathcal{F}, \mathcal{P})$, and $\mathbf{x}(t)$ is an unknown $m \times 1$ random vector.

We will assume that

$$
\boldsymbol{\xi}(t, \omega)=\boldsymbol{\xi}_{0}(t)+\varepsilon \boldsymbol{\xi}_{1}(t, \omega)+\varepsilon^{2} \boldsymbol{\xi}_{2}(t, \omega)+\cdots,
$$

where $\varepsilon$ is a parameter satisfying the condition $0 \leq \varepsilon \leq \varepsilon_{0}$. When $\varepsilon=0$ the problem $(1-1)$ reduces to the simple case

$$
\begin{gathered}
d \mathbf{x}(t) / d t=\mathbf{f}\left(t, \mathbf{x}(t), \boldsymbol{\xi}_{0}(t)\right), \\
\mathbf{A x}(0)+\mathbf{B} \mathbf{x}(1)=\boldsymbol{\alpha}(\omega) .
\end{gathered}
$$

${ }^{*}$ Received Sept. 11, 1986.

${ }^{1}$ The research described in this paper was carried out, in part, while the author was a Visiting Scholar at Rensselaer Polytechnic Institute, Troy, New York. 
Its solution $\mathbf{x}_{0}(t)$ can be found by means of the extended Liouville equation [10].

In the case that either matrix $\mathbf{A}$ or $\mathbf{B}$ is equal to zero, the problem (1-1) becomes an initial value problem. When $\varepsilon$ is small, its solution has a regular perturbation expansion, and the density function can be obtained by solving a sequence of firstorder deterministic partial differential equations; see $[3,11]$.

The main results of [11] can be extended to the boundary value problem (1-1). However, before we turn to a consideration of its expansion properties, we must first examine the existence of its solution. In fact, by the shooting method, as shown in Sec. 2, we can show that for every small $\varepsilon$ there exists a unique solution near $\mathbf{x}_{0}(t)$. As $\varepsilon \rightarrow 0$, a regular perturbation scheme is available; the solution of (1-1) and its density function have the asymptotic expansions

$$
\begin{aligned}
\mathbf{x}(t) & =\mathbf{x}_{0}(t)+\varepsilon \mathbf{x}_{1}(t)+\varepsilon^{2} \mathbf{x}_{2}(t)+\cdots, \\
p(\mathbf{x}, t) & =p_{0}(\mathbf{x}, t)+\varepsilon p_{1}(\mathbf{x}, t)+\varepsilon^{2} p_{2}(\mathbf{x}, t)+\cdots,
\end{aligned}
$$

which are established in Sec. 3.

To determine the density function, we introduce a new auxiliary vector stochastic process $\mathbf{z}(t)$. In the case that only the density function of $\mathbf{x}\left(t_{0}\right)$ is concerned, then

$$
\mathbf{z}(t)=\mathbf{A x}(T)+\mathbf{B} \mathbf{x}(t),
$$

where $T=(1-t) t_{0} /\left(1-t_{0}\right)$ and $0 \leq t_{0}<1$.

It is easy to see that $\mathbf{z}(t)$ has the properties

$$
\begin{gathered}
\mathbf{z}(1)=\mathbf{A x}(0)+\mathbf{B} \mathbf{x}(1)=\boldsymbol{\alpha}(\omega), \\
\mathbf{z}\left(t_{0}\right)=(\mathbf{A}+\mathbf{B}) \mathbf{x}\left(t_{0}\right) .
\end{gathered}
$$

If the density function of $\mathbf{z}(t)$ exists and is denoted by $p(\mathbf{z}, t)$, then

$$
p(\mathbf{z}, 1)=p_{\boldsymbol{\alpha}}(\mathbf{z}),
$$

and

$$
p_{\mathbf{x}\left(t_{0}\right)}(\mathbf{x})=P_{(\mathbf{A}+\mathbf{B})^{-1} \mathbf{z}\left(t_{0}\right)}(\mathbf{x})=\left.|\operatorname{Det}(\mathbf{A}+\mathbf{B})| P\left(\mathbf{z}, t_{0}\right)\right|_{\mathbf{z}=(\mathbf{A}+\mathbf{B}) \mathbf{x}},
$$

where $\mathbf{A}+\mathbf{B}$ is assumed to be not singular; $P_{\boldsymbol{\alpha}}(\cdot), P_{\mathbf{x}\left(t_{0}\right)}(\cdot)$, and $P_{(\mathbf{A}+\mathbf{B})-z^{z}\left(t_{0}\right)}(\cdot)$ are the density functions of $\boldsymbol{\alpha}(\omega), \mathbf{x}\left(t_{0}\right)$, and $(\mathbf{A}+\mathbf{B})^{-1} \mathbf{z}\left(t_{0}\right)$ respectively.

The condition (1-6) can be used to solve the first-order partial differential equation derived later, and (1-7) can be used to calculate the density function of $\mathbf{x}\left(t_{0}\right)$ if $p\left(\mathbf{z}, t_{0}\right)$ has been obtained.

If $0<t_{0} \leq 1$, we can obtain similar results by considering the auxiliary vector $\mathbf{y}(t)=\mathbf{A x}(t)+\mathbf{B x}\left(1+\left(1-\left(1 / t_{0}\right)\right) t\right)$ instead of $\mathbf{z}(t)$.

For simplicity, throughout this paper we will assume that $\mathbf{A}+\mathbf{B}$ is not singular, and only consider the properties of $\mathbf{z}(t)$. In the case that $\mathbf{A}+\mathbf{B}$ is singular, then instead of (1-3) we can let $\mathbf{w}(t)=\mathbf{A x}(T)+\left[\mathbf{B}+(1-t) \mathbf{B}^{*}\right] \mathbf{x}(t)$, where $\mathbf{B}^{*}$ is an auxiliary matrix to be determined. It is possible to show that $\mathbf{B}^{*}$ can always be chosen so that $\mathbf{A}+\mathbf{B}+\left(1-t_{0}\right) \mathbf{B}^{*}$ is not singular for $0 \leq t_{0}<1$. Then the density function of $\mathbf{x}\left(t_{0}\right)$ can be obtained by applying the method described in this paper to $w(t)$ instead of to $\mathbf{z}(t)$. For $0<t_{0} \leq 1$ we have a similar situation. 
As shown in $[7,8]$, under certain conditions, the density function $p(\mathbf{z}, t)$ must satisfy the partial differential equation

$$
\frac{\partial p(\mathbf{z}, t)}{\partial t}+\sum_{n_{1}+\cdots+n_{m}=1}^{\infty} \prod_{j=1}^{m} \frac{1}{\left(n_{j}\right) !} \frac{\partial^{n_{j}}}{\partial z_{j}^{n_{j}}}\left[\alpha_{n_{1}, \ldots, n_{m}}(\mathbf{z}, t) p(\mathbf{z}, t)\right]=0,
$$

where

$$
\alpha_{n_{1}, \ldots, n_{m}}(\mathbf{z}, t)=\lim _{\Delta t \rightarrow 0} \frac{1}{\Delta t} E\left\{\prod_{j=1}^{m}\left(z_{j}(t)-z_{j}(t-\Delta t)\right)^{n_{j}} \mid \mathbf{z}(t)=\mathbf{z}\right\},
$$

and $E\{\cdot \mid z(t)=\mathbf{z}\}$ denotes the mathematical expectation under the condition $\mathbf{z}(t)=\mathbf{z}$.

When $\varepsilon$ is small enough and only the terms up to order $\varepsilon^{2}$ are considered, the perturbation method reduces equation (1-8) to a first-order partial differential equation. The coefficients of this equation can be evaluated by means of some deterministic procedures and an auxiliary random problem. This is the topic of Sec. 4. In the last section, a simple example will be given to illustrate the whole procedure.

Compared with other existing numerical approximation methods $[3,5,6,9]$, the scheme presented here has the advantage of considerable generality. Also, one need not calculate higher-order coefficients if only a lower approximation is desired. In the case that the boundary value $\alpha$ is nonrandom, this method is still valid, but the delta function will occur in the derived first-order partial differential equation, which may be solved by using the Fourier transform. Furthermore, this expansion scheme can also be adapted for use on eigenvalue problems and on two-point boundary value problems containing white noise, which will be explored in other papers.

On the other hand, our method has the limitation of requiring the boundedness and smoothness of $\mathbf{f}$ and $\boldsymbol{\xi}$. Although extension of the time interval from $[0,1]$ to any finite interval $[0, M]$ is straightforward, no satisfactory results can be expected on arbitrarily long time intervals. As $t \rightarrow \infty$, other asymptotic schemes can be used if the processes involved in the problem have short correlation times, the boundary value is nonrandom, and the time has the order of $1 / \varepsilon$; see $[5,9]$.

2. Existence of the solution. For simplicity, in Secs. 2 and 3 we will only consider the scalar case, but the extension to higher dimensions is straightforward.

To prove the existence of the solution we define a random mapping and consider its fixed point, as outlined by White and Franklin [9] or Xia [12]. If $x_{0}(t, \alpha)$ is the solution of (1-2) and if

$$
J=A+B \exp \left[\int_{0}^{1} f_{x}\left(t, x_{0}(t, \alpha), \xi_{0}(t)\right) d t\right] \neq 0,
$$

then for every $\varepsilon>0$ we define a random mapping

$$
T^{\varepsilon}(\delta)=\delta-J^{-1}\left[A \delta+B y^{\varepsilon}(1, \delta)\right],
$$

where $y^{\varepsilon}(t, \delta)$ is the solution of the random initial value problem with the initial value $\delta(\omega)$,

$$
\left\{\begin{array}{l}
d y^{\varepsilon}(t, \delta) / d t=f\left(t, x_{0}(t, \alpha)+y^{\varepsilon}(t, \delta), \xi(t, \omega)\right)-f\left(t, x_{0}(t, \alpha), \xi_{0}(t)\right), \\
y^{\varepsilon}(0, \delta)=\delta(\omega) .
\end{array}\right.
$$


If we can prove that under certain assumptions $T^{\varepsilon}(\delta)$ is a contracting mapping defined on a suitable metric space, then by the well-known theorem there exists a unique random fixed point $\hat{\delta}$ such that $T^{\varepsilon}(\hat{\delta})=\hat{\delta}$, and thereby $x=x_{0}(t, \alpha)+y^{\varepsilon}(t, \hat{\delta})$ is the solution of (1-1). Unfortunately, this argument is not always valid for all $\omega \in \Omega$ in the general case, but when $\varepsilon$ is small we can show that it is true for all $\omega \in \mathcal{A}_{\Delta}^{\varepsilon}$, where the event $A_{\Delta}^{\varepsilon}$ has large probability and will be defined below. For fixed $\varepsilon, \Delta>0$, let

$$
\begin{aligned}
A_{\Delta}^{\varepsilon}=\left\{\omega \mid T^{\varepsilon}\right. \text { is a contracting mapping on the ball } \\
\text { of radius } \left.\Delta \text { about zero, with contraction } \frac{1}{2}\right\} ;
\end{aligned}
$$

that is, for all $\delta_{1}, \delta_{2}$ such that $\left|\delta_{i}\right| \leq \Delta, A_{\Delta}^{\varepsilon}$ is the event that $\left|T^{\varepsilon}\left(\delta_{i}\right)\right| \leq \Delta$, and $\left|T^{\varepsilon}\left(\delta_{1}\right)-T^{\varepsilon}\left(\delta_{2}\right)\right| \leq \frac{1}{2}\left|\delta_{1}-\delta_{2}\right|$.

THEOREM 1. Let $I=\{t \mid 0 \leq t \leq 1\}$, and suppose that

(a) $\xi(\cdot, \omega)$ is a continuous function, and $|\xi(t, \omega)| \leq K_{1}$ for $t \in I, \omega \in \Omega$;

(b) $E|\alpha(\omega)|^{2}<\infty$;

(c) there exists a solution $x_{0}(t)$ of Eq. (1-2), such that $E\left|x_{0}(0)\right|^{2}<\infty$ and $|J| \geq$ $K_{J}>0$;

(d) for $t \in I, x \in(-\infty, \infty),|\xi| \leq K_{1}, f, f_{x}, f_{x x}, f_{\xi}, f_{x \xi}, f_{\xi \xi}$ are continuous functions, and $|f(t, x, \xi)| \leq K_{2}(1+|x|),\left|f_{x}\right| \leq K_{3},\left|f_{\xi}\right| \leq K_{4},\left|f_{x x}\right| \leq K_{5},\left|f_{x \xi}\right| \leq K_{6}$, $\left|f_{\xi \xi}\right| \leq \bar{K}$, where $K_{J}, K_{1}, \cdots, K_{6}, \bar{K}$ are constants.

Then there exist two constants $\hat{\Delta}>0, \kappa>0$, independent of $\varepsilon$, such that

$$
\operatorname{prob}\left\{A_{\dot{\Delta}}^{\varepsilon}\right\} \geq 1-\kappa \varepsilon^{2}
$$

Proof. First we have the following estimations:

(a) $\quad\left|y^{\varepsilon}(t, \delta)\right| \leq K_{7}(\varepsilon+|\delta|)$,

(b) $\quad\left|y^{\varepsilon}\left(t, \delta_{2}\right)-y^{\varepsilon}\left(t, \delta_{1}\right)\right| \leq K_{8}\left|\delta_{2}-\delta_{1}\right|$,

(c) $\left|y^{\varepsilon}\left(t, \delta_{2}\right)-y^{\varepsilon}\left(t, \delta_{1}\right)-\left(\delta_{2}-\delta_{1}\right) \exp \left[\int_{0}^{t} f_{x}\left(t, x_{0}+y^{\varepsilon}\left(t, \delta_{1}\right), \xi\right) d t\right]\right|$

$$
\leq K_{9}\left(\delta_{2}-\delta_{1}\right)^{2},
$$

(d)

$$
\begin{aligned}
\mid \exp & {\left[\int_{0}^{1} f_{x}\left(t, x_{0}+y^{\varepsilon}(t, \delta), \xi\right) d t\right]-\exp \left[\int_{0}^{1} f_{x}\left(t, x_{0}, \xi_{0}\right) d t\right] \mid } \\
& \leq K_{10}\left(\varepsilon+|\delta|+\int_{0}^{1}\left|y^{\varepsilon}(t, 0)\right| d t\right) \\
& \leq K_{11}(\varepsilon+|\delta|),
\end{aligned}
$$


where $t \in I, \omega \in \Omega$, and $K_{7}, \ldots, K_{11}$ are suitable constants independent of $\varepsilon$.

To prove (2-5) we rewrite (2-2) in the integral form, note that $y^{\varepsilon}(t, \delta)$ is its solution, and use Gronwall's lemma. We then obtain $(2-5 a, b)$ easily.

For $(2-5 \mathrm{c})$, notice that

$$
\begin{aligned}
& \frac{d}{d t}\left[y^{\varepsilon}\left(t, \delta_{2}\right)-y^{\varepsilon}\left(t, \delta_{1}\right)-\left(\delta_{2}-\delta_{1}\right) \exp \left[\int_{0}^{t} f_{x}\left(t, x_{0}+y^{\varepsilon}\left(t, \delta_{1}\right), \xi\right) d t\right]\right] \\
&= f\left(t, x_{0}+y^{\varepsilon}\left(t, \delta_{2}\right), \xi\right)-f\left(t, x_{0}+y^{\varepsilon}\left(t, \delta_{1}\right), \xi\right) \\
&-\left(\delta_{2}-\delta_{1}\right) f_{x}\left(t, x_{0}+y^{\varepsilon}\left(t, \delta_{1}\right), \xi\right) \exp \left[\int_{0}^{t} f_{x}\left(t, x_{0}+y^{\varepsilon}\left(t, \delta_{1}\right), \xi\right) d t\right] \\
&= f_{x}\left(t, x_{0}+y^{\varepsilon}\left(t, \delta_{1}\right), \xi\right)\left[y^{\varepsilon}\left(t, \delta_{2}\right)-y^{\varepsilon}\left(t, \delta_{1}\right)\right]+\frac{f_{x x}(\lambda)}{2}\left[y^{\varepsilon}\left(t, \delta_{2}\right)-y^{\varepsilon}\left(t, \delta_{1}\right)\right]^{2} \\
&-\left(\delta_{2}-\delta_{1}\right) f_{x}\left(t, x_{0}+y^{\varepsilon}\left(t, \delta_{1}\right), \xi\right) \exp \left[\int_{0}^{t} f_{x}\left(t, x_{0}+y^{\varepsilon}\left(t, \delta_{1}\right), \xi\right) d t\right] \\
&= f_{x}\left(t, x_{0}+y^{\varepsilon}\left(t, \delta_{1}\right), \xi\right)\left[y^{\varepsilon}\left(t, \delta_{2}\right)-y^{\varepsilon}\left(t, \delta_{1}\right)-\left(\delta_{2}-\delta_{1}\right)\right. \\
&\left.\quad \times \exp \left[\int_{0}^{t} f_{x}\left(t, x_{0}+y^{\varepsilon}\left(t, \delta_{1}\right), \xi\right) d t\right]\right] \\
&+\frac{f_{x x}(\lambda)}{2}\left[y^{\varepsilon}\left(t, \delta_{2}\right)-y^{\varepsilon}\left(t, \delta_{1}\right)\right]^{2}, \quad
\end{aligned}
$$

where $f_{x x}(\lambda)=f_{x x}\left(t, x_{0}+y^{\varepsilon}\left(t, \delta_{1}\right)+\lambda\left(y^{\varepsilon}\left(t, \delta_{2}\right)-y^{\varepsilon}\left(t, \delta_{1}\right)\right)\right), \lambda \in[0,1]$; for simplicity we will use this notation in similar cases even for different $\lambda$. If we again change the above equation to its integral form and notice the boundedness assumption, then Gronwall's lemma can help us to obtain (2-5c) directly. The conclusion of (2-5d) can be proved in a similar way.

Now we turn to a consideration of $A_{\Delta}^{\varepsilon}$. Along the lines of White and Franklin [9], we define two events $B_{\Delta}^{\varepsilon}, C_{\Delta}^{\varepsilon}$ and try to prove that

$$
\operatorname{prob}\left\{\left(A_{\Delta}^{\varepsilon}\right)^{C}\right\} \leq \operatorname{prob}\left\{B_{\Delta}^{\varepsilon} \cup C_{\Delta}^{\varepsilon}\right\} \leq \kappa \varepsilon^{2},
$$

where

$$
\begin{aligned}
& B_{\Delta}^{\varepsilon}=\left\{\omega\left|\exists \delta_{0}:\right| \delta_{0}|\leq \Delta,| T^{\varepsilon}\left(\delta_{0}\right) \mid>\Delta\right\}, \\
& C_{\Delta}^{\varepsilon}=\left\{\omega\left|\exists \delta_{1}, \delta_{2}:\right| \delta_{1}|\leq \Delta,| \delta_{2}|\leq \Delta,| T^{\varepsilon}\left(\delta_{2}\right)-T^{\varepsilon}\left(\delta_{1}\right)\left|>\frac{1}{2}\right| \delta_{2}-\delta_{1} \mid\right\} .
\end{aligned}
$$

On the event $C_{\Delta}^{\varepsilon}$ we have $\delta_{1}, \delta_{2}$ such that $\left|\delta_{1}\right| \leq \Delta,\left|\delta_{2}\right| \leq \Delta$, and $\left|T^{\varepsilon}\left(\delta_{2}\right)-T^{\varepsilon}\left(\delta_{1}\right)\right|>$ $\frac{1}{2}\left|\delta_{2}-\delta_{1}\right|$. From the definition $(2-1)$ of $T^{\varepsilon}(\delta)$ we know that

$$
\begin{aligned}
\left|T^{\varepsilon}\left(\delta_{2}\right)-T^{\varepsilon}\left(\delta_{1}\right)\right| & =\left|\left(\delta_{2}-\delta_{1}\right)-J^{-1}\left[A\left(\delta_{2}-\delta_{1}\right)+B\left(y^{\varepsilon}\left(1, \delta_{2}\right)-y^{\varepsilon}\left(1, \delta_{1}\right)\right)\right]\right| \\
& \leq K_{J}^{-1}|B| \mid\left(\delta_{2}-\delta_{1}\right) \exp \left[\int_{0}^{1} f_{x}\left(t, x_{0}, \xi_{0}\right) d t\right] \\
& -\left(y^{\varepsilon}\left(1, \delta_{2}\right)-y^{\varepsilon}\left(1, \delta_{1}\right)\right) \mid .
\end{aligned}
$$


For $y^{\varepsilon}\left(t, \delta_{2}\right)-y^{\varepsilon}\left(t, \delta_{1}\right)$ we have the equation

$$
\begin{aligned}
& \frac{d}{d t}\left(y^{\varepsilon}\left(t, \delta_{2}\right)-y^{\varepsilon}\left(t, \delta_{1}\right)\right) \\
& \quad=f\left(t, x_{0}+y^{\varepsilon}\left(t, \delta_{2}\right), \xi\right)-f\left(t, x_{0}+y^{\varepsilon}\left(t, \delta_{1}\right), \xi\right) \\
& \quad=f_{x}\left(t, x_{0}+y^{\varepsilon}\left(t, \delta_{1}\right), \xi\right)\left(y^{\varepsilon}\left(t, \delta_{2}\right)-y^{\varepsilon}\left(t, \delta_{1}\right)\right)+\frac{f_{x x}(\lambda)}{2}\left(y^{\varepsilon}\left(t, \delta_{2}\right)-y^{\varepsilon}\left(t, \delta_{1}\right)\right)^{2} .
\end{aligned}
$$

It follows that

$$
\begin{aligned}
& y^{\varepsilon}\left(t, \delta_{2}\right)-y^{\varepsilon}\left(t, \delta_{1}\right)= \exp \left[\int_{0}^{t} f_{x}\left(t, x_{0}+y^{\varepsilon}\left(t, \delta_{1}\right), \xi\right) d t\right] \\
& \cdot\left\{\left(\delta_{2}-\delta_{1}\right)+\int_{0}^{t} \exp \left[-\int_{0}^{t} f_{x}\left(t, x_{0}+y^{\varepsilon}\left(t, \delta_{1}\right), \xi\right) d t\right]\right. \\
&\left.. \frac{f_{x x}(\lambda)}{2}\left(y^{\varepsilon}\left(t, \delta_{2}\right)-y^{\varepsilon}\left(t, \delta_{1}\right)\right)^{2} d t\right\} .
\end{aligned}
$$

Substituting this into (2-7) we obtain

$$
\begin{aligned}
\left|T^{\varepsilon}\left(\delta_{2}\right)-T^{\varepsilon}\left(\delta_{1}\right)\right| \leq K_{J}^{-1}|B|\{\mid & \left(\delta_{2}-\delta_{1}\right)\left(\exp \left[\int_{0}^{1} f_{x}\left(t, x_{0}+y^{\varepsilon}\left(t, \delta_{1}\right), \xi\right) d t\right]\right. \\
\left.-\exp \left[\int_{0}^{1} f_{x}\left(t, x_{0}, \xi_{0}\right) d t\right]\right) \mid & \\
& \left.+\left(K_{5} / 2\right) e^{2 K_{3}} \int_{0}^{1}\left|y^{\varepsilon}\left(t, \delta_{2}\right)-y^{\varepsilon}\left(t, \delta_{1}\right)\right|^{2} d t\right\} .
\end{aligned}
$$

From $(2-5 b, d)$ we have

$$
\begin{aligned}
& \left|T^{\varepsilon}\left(\delta_{2}\right)-T^{\varepsilon}\left(\delta_{1}\right)\right| \\
& \quad \leq K_{J}^{-1}|B|\left\{\left|\delta_{2}-\delta_{1}\right| K_{10}\left(\varepsilon+\left|\delta_{1}\right|+\int_{0}^{1}\left|y^{\varepsilon}(t, 0)\right| d t\right)+\frac{K_{5} K_{8}^{2}}{2} e^{2 K_{3}}\left(\delta_{2}-\delta_{1}\right)^{2}\right\} \\
& \leq K_{J}^{-1}|B| K_{12}\left(\varepsilon+\Delta+\int_{0}^{1}\left|y^{\varepsilon}(t, 0)\right| d t\right)\left|\delta_{2}-\delta_{1}\right|,
\end{aligned}
$$

where $K_{12}$ is a suitable constant independent of $\varepsilon$. Then on $C_{\Delta}^{\varepsilon}$ we know that

$$
\begin{aligned}
\frac{1}{2}\left|\delta_{2}-\delta_{1}\right| & <\left|T^{\varepsilon}\left(\delta_{2}\right)-T^{\varepsilon}\left(\delta_{1}\right)\right| \\
& \leq K_{J}^{-1}|B| K_{12}\left(\varepsilon+\Delta+\int_{0}^{1}\left|y^{\varepsilon}(t, 0)\right| d t\right)\left|\delta_{2}-\delta_{1}\right|,
\end{aligned}
$$

and then

$$
\frac{1}{2}<K_{J}^{-1}|B| K_{12}\left(\varepsilon+\Delta+\int_{0}^{1}\left|y^{\varepsilon}(t, 0)\right| d t\right) .
$$

Letting $K_{13}=\max \left\{1, K_{J}^{-1}|B| K_{12}\right\}$, we have

$$
\frac{1}{2} K_{13}^{-1}-\Delta<\varepsilon+\int_{0}^{1}\left|y^{\varepsilon}(t, 0)\right| d t
$$


Then we can always find $\Delta_{1}, l_{1}>0$, such that, when $0<\Delta \leq \Delta_{1}$,

$$
0<l_{1}<\varepsilon+\int_{0}^{1}\left|y^{\varepsilon}(t, 0)\right| d t .
$$

It follows that

$$
\begin{aligned}
\operatorname{prob}\left\{C_{\Delta}^{\varepsilon}\right\} & \leq \operatorname{prob}\left\{\omega\left|\varepsilon+\int_{0}^{1}\right| y^{\varepsilon}(t, 0) \mid d t>l_{1}>0\right\} \\
& \leq E\left[\varepsilon+\int_{0}^{1}\left|y^{\varepsilon}(t, 0)\right| d t\right]^{2} / l_{1}^{2} \leq\left(\left(1+K_{7}\right) / l_{1}\right)^{2} \varepsilon^{2}
\end{aligned}
$$

where $(2-5 a)$ has been used, and $\left(\left(1+K_{7}\right) / l_{1}\right)^{2}$ is independent of $\varepsilon$.

For the event $B_{\Delta}^{\varepsilon}$, we have $\delta_{0}$ such that $\left|\delta_{0}\right| \leq \Delta$, but $\left|T^{\varepsilon}\left(\delta_{0}\right)\right|>\Delta$. From the definition we know that

$$
\begin{aligned}
\left|T^{\varepsilon}\left(\delta_{0}\right)\right|= & \mid \delta_{0}-J^{-1}\left(A \delta_{0}+B y^{\varepsilon}\left(1, \delta_{0}\right) \mid\right. \\
\leq & K_{J}^{-1}|B|\left|\delta_{0} \exp \left[\int_{0}^{1} f_{x}\left(t, x_{0}, \xi_{0}\right) d t\right]-y^{\varepsilon}\left(1, \delta_{0}\right)\right| \\
\leq & K_{J}^{-1}|B|\left\{\left|y^{\varepsilon}(1,0)\right|+\mid y^{\varepsilon}\left(1, \delta_{0}\right)-y^{\varepsilon}(1,0)\right. \\
& \quad \delta_{0} \exp \left[\int_{0}^{1} f_{x}\left(t, x_{0}+y^{\varepsilon}(t, 0), \xi\right) d t\right] \mid \\
& +\left|\delta_{0}\right| \mid \exp \left[\int_{0}^{1} f_{x}\left(t, x_{0}+y^{\varepsilon}(t, 0), \xi\right) d t\right] \\
& \left.\quad-\exp \left[\int_{0}^{1} f_{x}\left(t, x_{0}, \xi_{0}\right) d t\right] \mid\right\} \\
\leq & K_{J}^{-1}|B|\left\{\left|y^{\varepsilon}(1,0)\right|+K_{9} \delta_{0}^{2}+K_{11} \varepsilon\left|\delta_{0}\right|\right\} \\
\leq & K_{14}\left\{\left|y^{\varepsilon}(1,0)\right|+\Delta^{2}+\varepsilon \Delta\right\},
\end{aligned}
$$

where $K_{14}>0$ is again a constant independent of $\varepsilon$. Let $\Delta_{2}$ be a constant such that $0<\Delta_{2} \leq \min \left\{1 /\left(2 K_{14}\right), \Delta_{1}\right\}$; then on event $B_{\Delta_{2}}^{\varepsilon}$ we have

$$
\Delta_{2}<\left|T^{\varepsilon}\left(\delta_{0}\right)\right| \leq K_{14}\left|y^{\varepsilon}(1,0)\right|+\left(\Delta_{2} / 2\right)+(\varepsilon / 2),
$$

or

$$
\left(\Delta_{2} / 2\right)<K_{14}\left|y^{\varepsilon}(1,0)\right|+(\varepsilon / 2)
$$

It follows that

$$
\begin{aligned}
\operatorname{prob}\left\{B_{\Delta_{2}}^{\varepsilon}\right\} & \leq \operatorname{prob}\left\{\omega\left|\left(\Delta_{2} / 2\right)<K_{14}\right| y^{\varepsilon}(1,0) \mid+(\varepsilon / 2)\right\} \\
& \leq \frac{4 E\left[K_{14}\left|y^{\varepsilon}(1,0)\right|+(\varepsilon / 2)\right]^{2}}{\Delta_{2}^{2}} \\
& \leq K_{15} \varepsilon^{2}
\end{aligned}
$$

where (2-5a) has been used again, and $K_{15}$ is a constant independent of $\varepsilon$.

Now let $\hat{\Delta}=\Delta_{2}$, and notice that $\left(A_{\hat{\Delta}}^{\varepsilon}\right)^{C} \subset B_{\hat{\Delta}}^{\varepsilon} \cup C_{\hat{\Delta}}^{\varepsilon}$; then we obtain (2-4) immediately. 
3. Asymptotic approximation. By the contracting mapping theorem, we know that there exists a unique fixed point $\hat{\delta}$ such that $T^{\varepsilon}(\hat{\delta})=\hat{\delta}$ for $\omega \in \mathcal{A}_{\hat{\Delta}}^{\varepsilon}$. To construct an approximate solution of equation (1-1), we define a random variable [12]

$$
\delta^{*}(\omega)= \begin{cases}\hat{\delta}, & \omega \in A_{\tilde{\Delta}}^{\varepsilon}, \\ \varepsilon x_{1}(0), & \omega \notin A_{\hat{\Delta}}^{\varepsilon},\end{cases}
$$

where $x_{1}(t)$ is the solution of the linear stochastic equation

$$
\left\{\begin{array}{l}
\frac{d x_{1}(t)}{d t}=f_{x}\left(t, x_{0}(t, \alpha), \xi_{0}(t)\right) x_{1}(t)+f_{\xi}\left(t, x_{0}(t, \alpha), \xi_{0}(t)\right) \xi_{1}(t, \omega), \\
A x_{1}(0)+B x_{1}(1)=0 .
\end{array}\right.
$$

Notice that by the existence of the solution $x_{1}(t)$, the random variable $\delta^{*}(\omega)$ is well defined. Moreover, we have the following lemma:

Lemma 1. Let all the assumptions of Theorem 1 be satisfied. Then there exists a constant $C$, independent of $\varepsilon$, such that

$$
E\left[\left(\delta^{*}(\omega)\right)^{2 n}\right] \leq C \varepsilon^{2 n} \text { for } n=1,2, \ldots
$$

Proof. Let $\chi_{A_{\dot{\Delta}}^{\varepsilon}}$ be the characteristic function of $A_{\hat{\Delta}}^{\varepsilon}$, i.e.,

$$
\chi_{A_{\dot{\Delta}}^{\varepsilon}}(\omega)= \begin{cases}1, & \omega \in A_{\bar{\Delta}^{\prime}}^{\varepsilon} \\ 0, & \text { otherwise. }\end{cases}
$$

By the definition of $\delta^{*}$ and $\AA_{\hat{\Delta}}^{\varepsilon}$, we have

$$
\left|T^{\varepsilon}\left(\delta^{*}\right)-T^{\varepsilon}(0)\right| \chi_{A_{j}^{\varepsilon}}=\left|\delta^{*}-T^{\varepsilon}(0)\right| \chi_{A_{\dot{\Delta}}^{\varepsilon}}
$$

and

It follows that

$$
\left|T^{\varepsilon}\left(\delta^{*}\right)-T^{\varepsilon}(0)\right| \chi_{A_{\dot{\Delta}}^{\varepsilon}} \leq \frac{1}{2}\left|\delta^{*}-0\right| \chi_{A_{\dot{\Delta}}^{\varepsilon}}=\frac{1}{2}\left|\delta^{*}\right| \chi_{A_{\dot{\alpha}}^{\varepsilon}} .
$$

$$
\left|\delta^{*}-T^{\varepsilon}(0)\right| \chi_{A_{\dot{\delta}}^{\varepsilon}} \leq \frac{1}{2}\left|\delta^{*}\right| \chi_{A_{\dot{\Delta}}^{\varepsilon}}
$$

or

$$
\left|\delta^{*}\right| \chi_{A_{\dot{\Delta}}^{\varepsilon}} \leq 2\left|T^{\varepsilon}(0)\right| \chi_{A_{\dot{\Delta}}^{\varepsilon}} \leq 2 K_{J}^{-1}|B|\left|y^{\varepsilon}(1,0)\right| .
$$

Then from $(2-5 \mathrm{a})$ we have

$$
\begin{aligned}
E\left[\left|\delta^{*}\right|^{2 n} \chi_{A_{\Delta}^{\varepsilon}}\right] & \leq\left(2 K_{J}^{-1}|B|\right)^{2 n} E\left[\left|y^{\varepsilon}(1,0)\right|^{2 n}\right] \\
& \leq\left(2 K_{J}^{-1}|B| K_{7}\right)^{2 n} \varepsilon^{2 n} .
\end{aligned}
$$

To estimate $\delta^{*}$ for $\omega \notin \mathcal{A}_{\hat{\Delta}}^{\varepsilon}$, we solve the linear two-point boundary value problem (3-2) and find that

$$
\begin{aligned}
x_{1}(t)=\exp \left[\int_{0}^{t} f_{x}\left(t, x_{0}(t, \alpha), \xi_{0}\right) d t\right] \\
\cdot\left\{\int_{0}^{t} \exp \left[-\int_{0}^{s} f_{x}\left(s, x_{0}(s, \alpha), \xi_{0}\right) d s\right] f_{\xi}\left(s, x_{0}(s, \alpha), \xi_{0}\right) \xi_{1}(s, \omega) d s\right. \\
\quad-J^{-1} B \exp \left[\int_{0}^{1} f_{x}\left(t, x_{0}(t, \alpha), \xi_{0}\right) d t\right] \\
\left.\cdot \int_{0}^{1} \exp \left[-\int_{0}^{s} f_{x}\left(s, x_{0}(s, \alpha), \xi_{0}\right) d s\right] f_{\xi}\left(s, x_{0}(s, \alpha), \xi_{0}\right) \xi_{1}(s, \omega) d s\right\} .
\end{aligned}
$$


Then

$$
\begin{aligned}
\left|\delta^{*}\right|^{2 n}\left(1-\chi_{A_{\dot{\Delta}}^{\varepsilon}}\right)= & \varepsilon^{2 n} \mid J^{-1} B \exp \left[\int_{0}^{1} f_{x}\left(t, x_{0}, \xi_{0}\right) d t\right] \\
& \left.\cdot \int_{0}^{1} \exp \left[-\int_{0}^{s} f_{x}\left(s, x_{0}, \xi_{0}\right) d s\right] f_{\xi}\left(s, x_{0}, \xi_{0}\right) \xi_{1}(s, \omega) d s\right|^{2 n}\left(1-\chi_{A_{\dot{\delta}}^{\varepsilon}}\right),
\end{aligned}
$$

and its expectation satisfies

$$
E\left[\left|\delta^{*}\right|^{2 n}\left(1-\chi_{\beta_{\dot{\Delta}}^{c}}\right)\right] \leq C^{*} \varepsilon^{2 n},
$$

where the constant $C^{*}$ is independent of $\varepsilon$, and the boundedness assumptions on $f_{x}$, $J^{-1}, f_{\xi}, \xi_{1}$ have been used again. Combining (3-4) with (3-5), we find that

$$
E\left[\left|\delta^{*}\right|^{2 n}\right]=E\left[\left|\delta^{*}\right|^{2 n} \chi_{A_{\dot{\Delta}}^{\varepsilon}}\right]+E\left[\left|\delta^{*}\right|^{2 n}\left(1-\chi_{A_{\dot{\Delta}}^{\varepsilon}}\right)\right] \leq C \varepsilon^{2 n},
$$

thus completing the proof.

Now we are in a position to consider the asymptotic property of the solution of Eq. (1-1). Notice that because $A_{\hat{\Delta}}^{\varepsilon}$ has large probability as $\varepsilon \rightarrow 0$, we can use the random process $x=x_{0}(t, \alpha)+y^{\varepsilon}\left(t, \delta^{*}\right)$ as the approximate solution. We can prove that it has the expansion

$$
x(t)=x_{0}(t)+\varepsilon x_{1}(t)+\varepsilon^{2} x_{2}(t)+\cdots
$$

where $[\cdots]$ is a quantity with order greater than $\varepsilon^{2}, x_{0}, x_{1}$ are the functions mentioned before, and $x_{2}(t)$ is the solution of the following linear stochastic equation:

$$
\left\{\begin{aligned}
\frac{d x_{2}(t)}{d t}=f_{x}\left(t, x_{0}, \xi_{0}\right) x_{2}(t)+ & {\left[f_{\xi}\left(t, x_{0}, \xi_{0}\right) \xi_{2}+\frac{f_{x x}\left(t, x_{0}, \xi_{0}\right)}{2} x_{1}^{2}\right.} \\
& \left.+f_{x \xi}\left(t, x_{0}, \xi_{0}\right) x_{1} \xi_{1}+\frac{f_{\xi \xi}\left(t, x_{0}, \xi_{0}\right)}{2} \xi_{1}^{2}\right], \\
A x_{2}(0)+B x_{2}(1)=0 . &
\end{aligned}\right.
$$

For $\varepsilon=0,(3-6)$ is valid, so we need consider only the case when $\varepsilon \neq 0$.

THEOREM 2. Let all the hypotheses of Theorem 1 be satisfied. Then there exists a constant $K_{16}$, independent of $\varepsilon$, such that

$$
\sup _{t \in I} E\left[\left(x-x_{0}\right) / \varepsilon-x_{1}\right]^{2} \leq K_{16} \varepsilon^{2} .
$$

Proof. Since $x=x_{0}+y^{\varepsilon}\left(t, \delta^{*}\right)$, we have the following equation for $\left(y^{\varepsilon} / \varepsilon\right)-x_{1}$ :

$$
\left\{\begin{array}{rlrl}
\frac{d\left(y^{\varepsilon} / \varepsilon-x_{1}\right)}{d t}=[ & \left.f\left(t, x_{0}+y^{\varepsilon}, \xi\right)-f\left(t, x_{0}, \xi_{0}\right)\right] / \varepsilon & & \\
- & {\left[f_{x}\left(t, x_{0}, \xi_{0}\right) x_{1}+f_{\xi}\left(t, x_{0}, \xi_{0}\right) \xi_{1}\right]} & \\
\left.A\left(y^{\varepsilon} / \varepsilon-x_{1}\right)\right|_{t=0}+\left.B\left(y^{\varepsilon} / \varepsilon-x_{1}\right)\right|_{t=1}=0, & & \omega \in A_{\hat{\Delta}}^{\varepsilon} \\
\left.\left(y^{\varepsilon} / \varepsilon-x_{1}\right)\right|_{t=0}=0, & \omega \notin A_{\tilde{\Delta}}^{\varepsilon} .
\end{array}\right.
$$


We expand the functions in Eq. (3-9); then

$$
\begin{aligned}
\frac{d\left(y^{\varepsilon} / \varepsilon-x_{1}\right)}{d t}= & f_{x}\left(t, x_{0}, \xi_{0}\right)\left(y^{\varepsilon} / \varepsilon-x_{1}\right) \\
& +\left[f_{\xi}\left(t, x_{0}, \xi_{0}\right)\left(\left(\xi-\xi_{0}\right) / \varepsilon-\xi_{1}\right)+\left(f_{x x}(\lambda) / 2\right)\left(y^{\varepsilon} / \varepsilon\right)^{2} \varepsilon\right. \\
& \left.\quad+f_{x \xi}(\lambda)\left(y^{\varepsilon} / \varepsilon\right)\left(\xi-\xi_{0}\right)+\left(f_{\xi \xi}(\lambda) / 2\right)\left(\left(\xi-\xi_{0}\right)^{2} / \varepsilon\right)\right] .
\end{aligned}
$$

On $A_{\hat{\Delta}}^{\varepsilon}$, we have

$$
\begin{aligned}
\left(y^{\varepsilon} / \varepsilon\right)-x_{1}= & \varepsilon \exp \left[\int_{0}^{t} f_{x}\left(t, x_{0}, \xi_{0}\right) d t\right] \\
& \cdot\left[\int_{0}^{t} F(s) \exp \left[-\int_{0}^{s} f_{x}\left(s, x_{0}, \xi_{0}\right) d s\right] d s\right. \\
& -J^{-1} B \exp \left[\int_{0}^{1} f_{x}\left(t, x_{0}, \xi_{0}\right) d t\right] \\
& \left.\cdot \int_{0}^{1} F(s) \exp \left[-\int_{0}^{s} f_{x}\left(s, x_{0}, \xi_{0}\right) d s\right] d s\right],
\end{aligned}
$$

where

$$
\begin{aligned}
F(s)= & \frac{1}{\varepsilon} f_{\xi}\left(s, x_{0}, \xi_{0}\right)\left(\left(\xi-\xi_{0}\right) / \varepsilon-\xi_{1}\right)+\left(f_{x x}(\lambda) / 2\right)\left(y^{\varepsilon} / \varepsilon\right)^{2} \\
& +f_{x \xi}(\lambda)\left(y^{\varepsilon} / \varepsilon\right)\left(\xi-\xi_{0}\right) / \varepsilon+\left(f_{\xi \xi}(\lambda) / 2\right)\left(\left(\xi-\xi_{0}\right) / \varepsilon\right)^{2} .
\end{aligned}
$$

By means of Lemma 1, from (2-5a) we easily find the estimate

$$
\sup _{t \in I} E\left[\left(\frac{y^{\varepsilon}\left(t, \delta^{*}\right)}{\varepsilon}\right)^{2 n}\right] \leq K_{17}, \quad n=1,2,
$$

with the constant $K_{17}$ independent of $\varepsilon$. Substituting it into (3-10) and noticing the boundedness assumptions on $f_{x}, f_{\xi}, f_{x x}, f_{x \xi}, f_{\xi \xi}$, we obtain

$$
\sup _{t \in I} E\left\{\left[\frac{y^{\varepsilon}\left(t, \delta^{*}\right)}{\varepsilon}-x_{1}\right]^{2} \chi_{A_{\dot{\Delta}}^{\varepsilon}}\right\} \leq K_{18} \varepsilon^{2} .
$$

When $\omega \notin A_{\hat{\Delta}}^{\varepsilon}$, the initial value problem (3-9) gives

$$
\frac{y^{\varepsilon}\left(t, \delta^{*}\right)}{\varepsilon}-x_{1}=\varepsilon \exp \left[\int_{0}^{t} f_{x}\left(t, x_{0}, \xi_{0}\right) d t\right] \int_{0}^{t} F(s) \exp \left[-\int_{0}^{s} f_{x}\left(s, x_{0}, \xi_{0}\right) d s\right] d s,
$$

and then we have the estimate

$$
\sup _{t \in I} E\left\{\left[\frac{y^{\varepsilon}\left(t, \delta^{*}\right)}{\varepsilon}-x_{1}\right]^{2}\left(1-\chi_{\beta_{\dot{\Delta}}^{\varepsilon}}\right)\right\} \leq K_{19} \varepsilon^{2} .
$$

Combining (3-12) and (3-13) and noticing that $K_{18}, K_{19}$ are independent of $\varepsilon$, we obtain (3-9) and thus complete the proof.

In a similar way we can establish the quadratic approximation (3-6), or more precisely 
THEOREM 3. Let all the assumptions of Theorem 1 be satisfied, and suppose that the derivatives $f_{x x x}, f_{x x \xi}, f_{x \xi \xi}, f_{\xi \xi \xi}$ are bounded continuous functions for $t \in I$, $x \in(-\infty, \infty)$, and $|\xi| \leq K_{1}$; then as $\varepsilon \rightarrow 0$ we have

$$
\sup _{t \in I} E\left[\left(x-x_{0}-\varepsilon x_{1}\right) / \varepsilon^{2}-x_{2}\right]^{2} \rightarrow 0,
$$

where $x_{2}$ is the solution of the linear problem (3-7).

4. Density function of the solution. From the fact that $\mathbf{x}(t)$ has a regular perturbation approximation as $\varepsilon \rightarrow 0$, we know that $\mathbf{z}(t)$ and its density function $p(\mathbf{z}, t)$ can be expanded also in regular forms. This simplifies the partial differential equation (1-8), which is satisfied by $p(\mathbf{z}, t)$, and makes it accessible. The coefficients of equation (1-8) are given by the formula (1-9). To evaluate them we must use the condition $\mathbf{z}(t)=\mathbf{z}$. In order to avoid possible confusion, we introduce a new time variable $t^{*}$, so that the condition becomes

$$
\left.\mathbf{z}\left(t^{*}\right)\right|_{t^{*}=t}=\mathbf{z} \text { or }\left.\quad \mathbf{A x}\left(t^{*}\right)\right|_{t^{*}=T}+\left.\mathbf{B x}\left(t^{*}\right)\right|_{t^{*}=t}=\mathbf{z} .
$$

If only the terms up to order $\varepsilon$ are considered, then under the given condition we know that $\mathbf{x}\left(t^{*}\right)=\mathbf{x}_{0}\left(t^{*}\right)+\varepsilon \mathbf{x}_{1}\left(t^{*}\right)+O\left(\varepsilon^{2}\right)$, where $\mathbf{x}_{0}\left(t^{*}\right)$ and $\mathbf{x}_{1}\left(t^{*}\right)$ satisfy the following equations:

$$
\left\{\begin{array}{l}
\frac{d \mathbf{x}_{0}\left(l^{*}\right)}{d t^{*}}=\mathbf{f}\left(t^{*}, \mathbf{x}_{0}\left(t^{*}\right), \boldsymbol{\xi}_{0}\left(t^{*}\right)\right) \\
\mathbf{A x}_{0}(T)+\mathbf{B} \mathbf{x}_{0}(t)=\mathbf{z}
\end{array}\right.
$$

and

$$
\left\{\begin{array}{l}
\frac{d \mathbf{x}_{1}\left(t^{*}\right)}{d t^{*}}=\mathbf{f}_{x}\left(t^{*}, \mathbf{x}_{0}\left(t^{*}\right), \boldsymbol{\xi}_{0}\left(t^{*}\right)\right) \mathbf{x}_{1}\left(t^{*}\right)+\mathbf{f}_{\xi}\left(t^{*}, \mathbf{x}_{0}\left(t^{*}\right), \boldsymbol{\xi}_{0}\left(t^{*}\right)\right) \boldsymbol{\xi}_{1}\left(t^{*}, \omega\right) \\
\mathbf{A} \mathbf{x}_{1}(T)+\mathbf{B} \mathbf{x}_{1}(t)=0
\end{array}\right.
$$

where the regular perturbation method is used both for the differential equation and the condition $\left.\mathbf{z}\left(t^{*}\right)\right|_{t^{*}=t}=\mathbf{z}$.

For every deterministic vector $\mathbf{z}$, the problem (4-1) is deterministic, and its solution can be found by deterministic procedures. To emphasize the dependence on $t$ and $\mathbf{z}$, we denote the solution of (4-1) by $\mathbf{x}_{0}\left(t^{*}\right)=\mathbf{x}_{0}\left(t^{*}, \mathbf{z}, t\right)$.

The problem (4-2) is linear and random, and its solution is

$$
\begin{aligned}
\mathbf{x}_{1}\left(t^{*}\right)=\psi\left(t^{*}\right)\left\{\int_{T}^{t^{*}} \psi^{-1}(s) \mathbf{f}_{\xi}\left(s, \mathbf{x}_{0}(s, \mathbf{z}, t), \boldsymbol{\xi}_{0}(s)\right) \boldsymbol{\xi}_{1}(s, \omega) d s\right. \\
\left.-\mathbf{B} \psi(t) \int_{T}^{t} \psi^{-1}(s) \mathbf{f}_{\xi}\left(s, \mathbf{x}_{0}(s, \mathbf{z}, t) \boldsymbol{\xi}_{0}(s)\right) \boldsymbol{\xi}_{1}(s, \omega) d s\right\}
\end{aligned}
$$

where $\psi\left(t^{*}\right)$ is the fundamental matrix of the problem

$$
\left\{\begin{array}{l}
\frac{d \psi\left(t^{*}\right)}{d t^{*}}=\mathbf{f}_{x}\left(t^{*}, \mathbf{x}_{0}\left(t^{*}, \mathbf{z}, t\right), \boldsymbol{\xi}_{0}\left(t^{*}\right)\right) \psi\left(t^{*}\right) \\
\mathbf{A} \psi(T)+\mathbf{B} \psi(t)=\mathbf{I}
\end{array}\right.
$$

and $\mathbf{I}$ is the identity matrix. 
The condition $\mathbf{z}(t)=\mathbf{z}$ also implies a restriction on $\alpha(\omega)$; in fact, since $\alpha(\omega)=$ $\mathbf{A x}(0)+\mathbf{B x}(1)$, then under the given condition, we have

$$
\begin{aligned}
\boldsymbol{\alpha}(\omega)= & \mathbf{A}\left[\left.\mathbf{x}_{0}\left(t^{*}, \mathbf{z}, t\right)\right|_{t^{*}=0}+\left.\varepsilon \mathbf{x}_{1}\left(t^{*}, \mathbf{z}, t\right)\right|_{t^{*}=0}+O\left(\varepsilon^{2}\right)\right] \\
& +\mathbf{B}\left[\left.\mathbf{x}_{0}\left(t^{*}, \mathbf{z}, t\right)\right|_{t^{*}=1}+\left.\varepsilon \mathbf{x}_{1}\left(t^{*}, \mathbf{z}, t\right)\right|_{t^{*}=1}+O\left(\varepsilon^{2}\right)\right] \\
= & \mathbf{A x}_{0}(0, \mathbf{z}, t)+\mathbf{B} \mathbf{x}_{0}(1, \mathbf{z}, t)+O(\varepsilon) \\
= & q(\mathbf{z}, t)+O(\varepsilon),
\end{aligned}
$$

where $q(\mathbf{z}, t)=\mathbf{A x}_{0}(0, \mathbf{z}, t)+\mathbf{B} \mathbf{x}_{0}(1, \mathbf{z}, t)$.

Now we consider the term $z_{j}(t)-z_{j}(t-\Delta t)$ in the coefficients (1-9). From the definition (1-3) of $\mathbf{z}(t)$, and the equation (1-1a), we have

$$
\begin{aligned}
z_{j}(t)-z_{j}(t-\Delta t) & =\left[\mathbf{A}_{j} \mathbf{x}(T)+\mathbf{B}_{j} \mathbf{x}(t)\right]_{t}^{\prime} \Delta t+O\left(\Delta t^{2}\right) \\
& =\left(D_{j}+\varepsilon H_{j}\right) \Delta t+O\left(\Delta t^{2}\right)+O\left(\varepsilon^{2}\right)
\end{aligned}
$$

where $\mathbf{A}_{j}, \mathbf{B}_{j}$ are the $j$ th row vectors of the matrices $\mathbf{A}, \mathbf{B}$ respectively, and

$$
\begin{aligned}
D_{j}= & \mathbf{A}_{j}\left(-t_{0} /\left(1-t_{0}\right)\right) \mathbf{f}\left(T, \mathbf{x}_{0}(T), \boldsymbol{\xi}_{0}(T)\right)+\mathbf{B}_{j} \mathbf{f}\left(t, \mathbf{x}_{0}(t), \boldsymbol{\xi}_{0}(t)\right) \\
H_{j}= & \mathbf{A}_{j}\left(-t_{0} /\left(1-t_{0}\right)\right)\left[\mathbf{f}_{x}\left(T, \mathbf{x}_{0}(T), \boldsymbol{\xi}_{0}(T)\right) \mathbf{x}_{1}(T)+\mathbf{f}_{\xi}\left(T, \mathbf{x}_{0}(T), \boldsymbol{\xi}_{0}(T)\right) \boldsymbol{\xi}_{1}(T, \omega)\right] \\
& +\mathbf{B}_{j}\left[\mathbf{f}_{x}\left(t, \mathbf{x}_{0}(t), \boldsymbol{\xi}_{0}(t)\right) \mathbf{x}_{1}(t)+\mathbf{f}_{\xi}\left(t, \mathbf{x}_{0}(t), \boldsymbol{\xi}_{0}(t)\right) \boldsymbol{\xi}_{1}(t, \omega)\right]
\end{aligned}
$$

Then

$$
\left[z_{j}(t)-z_{j}(t-\Delta t)\right]^{n_{j}}=\left[D_{j}^{n_{j}}+\varepsilon n_{j} D_{j}^{n_{j}-1} H_{j}\right] \Delta t^{n_{j}}+O\left(\Delta t^{n_{j}+1}\right)+O\left(\varepsilon^{2}\right)
$$

and

$$
\begin{aligned}
\prod_{j=1}^{m}\left[z_{j}(t)-z_{j}(t-\Delta t)\right]^{n_{j}} \\
=\left\{\prod_{j=1}^{m} D_{j}^{n_{j}}+\varepsilon\left[n_{1} D_{1}^{n_{1}-1} D_{2}^{n_{2}} \cdots D_{m}^{n_{m}} H_{1}+\cdots\right.\right. \\
\left.\left.+n_{m} D_{1}^{n_{1}} D_{2}^{n_{2}} \cdots D_{m}^{n_{m}-1} H_{m}\right]\right\}(\Delta t)^{\sum_{j=1}^{m} n_{j}} \\
+O\left(\Delta t^{\left(\sum_{j=1}^{m} n_{j}\right)+1}\right)+O\left(\varepsilon^{2}\right) .
\end{aligned}
$$

Under the condition $\mathbf{z}(t)=\mathbf{z}$, we know that $D_{j}$ depends on the deterministic function 
$\mathbf{x}_{0}\left(t^{*}, \mathbf{z}, t\right)$, so that

$$
\begin{aligned}
& E\left\{\prod_{j=1}^{m}\left[z_{j}(t)-z_{j}(t-\Delta t)\right]^{n_{j}} \mid \mathbf{z}(t)=\mathbf{z}\right\} \\
& =\left\{\left.\prod_{j=1}^{m} D_{j}^{n_{j}}\right|_{\substack{\mathbf{x}_{0}(T)=\mathbf{x}_{0}(T, \mathbf{z}, t) \\
\mathbf{x}_{0}(t)=\mathbf{x}_{0}(t, \mathbf{z}, t)}}+\varepsilon\left\{\left.E\left[H_{1} \mid \mathbf{z}(t)=\mathbf{z}\right]\left[n_{1} D_{1}^{n_{1}-1} D_{2}^{n_{2}} \cdots D_{m}^{n_{m}}\right]\right|_{\substack{\mathbf{x}_{0}(T)=\mathbf{x}_{0}(T, \mathbf{z}, t) \\
\mathbf{x}_{0}(t)=\mathbf{x}_{0}(t, \mathbf{z}, t)}}\right.\right. \\
& \left.\left.+\cdots+\left.E\left[H_{m} \mid \mathbf{z}(t)=\mathbf{z}\right]\left[n_{m} D_{1}^{n_{1}} D_{2}^{n_{2}} \cdots D_{m}^{n_{m}-1}\right]\right|_{\substack{\mathbf{x}_{0}(T)=\mathbf{x}_{0}(T, \mathbf{z}, t) \\
\mathbf{x}_{0}(t)=\mathbf{x}_{0}(t, \mathbf{z}, t)}}\right\}\right\}(\Delta t)^{\sum_{j=1}^{m} n_{j}} \\
& \quad+O\left(\Delta t^{\left(\sum_{j=1}^{m} n_{j}\right)+1}\right)+O\left(\varepsilon^{2}\right) .
\end{aligned}
$$

Notice that if we keep only the terms of order up to $\varepsilon$, then to evaluate (4-10) we now need to calculate only the main part of $E\left[H_{j} \mid \mathbf{z}(t)=\mathbf{z}\right]$, that is,

$$
\begin{aligned}
& E\left[H_{j} \mid \mathbf{z}(t)=\mathbf{z}\right]=\mathbf{A}_{j}\left(-t_{0} /\left(1-t_{0}\right)\right)\left\{\mathbf{f}_{x}\left(T, \mathbf{x}_{0}(T, \mathbf{z}, t), \boldsymbol{\xi}_{0}(T)\right)\right. \\
& \psi \psi(T)\left[-\mathbf{B} \psi(t) \int_{T}^{t} \psi^{-1}(s) \mathbf{f}_{\xi}\left(s, \mathbf{x}_{0}(s, \mathbf{z}, t), \boldsymbol{\xi}_{0}(s)\right) E\left[\psi_{1}(s, \omega) \mid \boldsymbol{\alpha}(\omega)=\mathbf{q}(\mathbf{z}, t)\right] d s\right] \\
&\left.+\mathbf{f}_{\psi}\left(T, \mathbf{x}_{0}(T, \mathbf{z}, t), \psi_{0}(T)\right) E\left[\boldsymbol{\xi}_{1}(T, \omega) \mid \boldsymbol{\alpha}(\omega)=\mathbf{q}(\mathbf{z}, t)\right]\right\} \\
&+\mathbf{B}_{j}\left\{\mathbf{f}_{x}\left(t, \mathbf{x}_{0}(t, \mathbf{z}, t), \boldsymbol{\xi}_{0}(t)\right) \psi(t)\right. \\
&+\left[\mathbf{A} \psi(T) \int_{T}^{t} \psi^{-1}(s) \mathbf{f}_{\xi}\left(s, \mathbf{x}_{0}(s, \mathbf{z}, t), \boldsymbol{\xi}_{0}(s)\right) E\left[\boldsymbol{\xi}_{1}(s, \omega) \mid \boldsymbol{\alpha}(\omega)=\mathbf{q}(\mathbf{z}, t)\right] d s\right] \\
&\left.+\mathbf{f}_{\xi}\left(t, \mathbf{x}_{0}(t, \mathbf{z}, t), \boldsymbol{\xi}_{0}(t)\right) E\left[\boldsymbol{\xi}_{1}(t, \omega) \mid \boldsymbol{\alpha}(\omega)=\mathbf{q}(\mathbf{z}, t)\right]\right\}+O(\varepsilon),
\end{aligned}
$$

where (4-8), (4-3), and (4-5) have been used. The term

$$
E\left[\xi_{1}(t, \omega) \mid \boldsymbol{\alpha}(\omega)=\mathbf{q}(\mathbf{z}, t)\right]=\left.\frac{\int \boldsymbol{\xi}_{1} p_{\boldsymbol{\alpha} \xi_{1}(t)}\left(\boldsymbol{\alpha}, \boldsymbol{\xi}_{1}\right) d \xi_{1}}{\int p_{\boldsymbol{\alpha} \xi_{1}(t)}\left(\boldsymbol{\alpha}, \boldsymbol{\xi}_{1}\right) d \boldsymbol{\xi}_{1}}\right|_{\boldsymbol{\alpha}=\mathbf{q}(\mathbf{z}, t)}
$$

can be evaluated in terms of the joint density function $p_{\alpha \xi_{1}(t)}$ of the random vectors $\alpha(\omega), \xi_{1}(t, \omega)$; and we always assume that (4-12) is bounded.

Substituting (4-10) into (1-9), we find that the coefficients have the form

$$
\begin{aligned}
\alpha_{n_{1}, \ldots, n_{m}}(\mathbf{z}, t) & =\lim _{\Delta t \rightarrow 0} \frac{1}{\Delta t} E\left\{\left[\prod_{j=1}^{m}\left(z_{j}(t)-z_{j}(t-\Delta t)\right)^{n_{j}}\right] \mid \mathbf{z}(t)=\mathbf{z}\right\} \\
& =\lim _{\Delta t \rightarrow 0}\left\{[\cdots] \Delta t^{\left(\sum_{j=1}^{m} n_{j}\right)-1}+O\left(\Delta t^{\left(\sum_{j=1}^{m} n_{j}\right)}\right)+O\left(\varepsilon^{2}\right)\right\}
\end{aligned}
$$


where $[\cdots]$ is a bounded quantity. Omitting the $\varepsilon^{2}$ term, we then find that $\alpha_{n_{1}, \ldots, n_{m}}(\mathbf{z}, t)$ $=0$, unless $n_{1}+\cdots+n_{m}=1$, that is, unless

$$
n_{j}= \begin{cases}1, & \text { for some } j \\ 0, & \text { otherwise }\end{cases}
$$

In this case we let $\alpha_{j}(\mathbf{z}, t)=\alpha_{0, \ldots, 1, \ldots, 0}(\mathbf{z}, t)$, where the 1 is in the $j$ th spot. The partial differential equation (1-8) now can be reduced to the first-order equation

$$
\frac{\partial p(\mathbf{z}, t)}{\partial t}+\sum_{j=1}^{m} \frac{\partial}{\partial z_{j}}\left[\alpha_{j}(\mathbf{z}, t) p(\mathbf{z}, t)\right]=0 .
$$

The coefficients $\alpha_{j}(\mathbf{z}, t)$ can be obtained by the procedure discussed above, and the solution of (4-13) can be found under the condition (1-6).

The numerical procedure for evaluating the density function of the solution of equation (1-1) up to order $\varepsilon$ is as follows:

(a) For fixed $\mathbf{z}, t$ solve the problem (4-1) to obtain the solution $\mathbf{x}_{0}\left(t^{*}\right)=\mathbf{x}_{0}\left(t^{*}, \mathbf{z}, t\right)$.

(b) Use (4-4), (4-5), (4-12), and (4-11) to evaluate $E\left[H_{j} \mid \mathbf{z}(t)=\mathbf{z}\right]$.

(c) Use (4-7) to evaluate $E\left[D_{j} \mid \mathbf{z}(t)=\mathbf{z}\right]$.

(d) Solve the following first-order partial differential equations:

$$
\left\{\begin{array}{l}
\frac{\partial p_{0}(\mathbf{z}, t)}{\partial t}+\sum_{j=1}^{m} \frac{\partial}{\partial z}\left\{E\left[D_{j} \mid \mathbf{z}(t)=\mathbf{z}\right] p_{0}(\mathbf{z}, t)\right\}=0, \\
\left.p_{0}(\mathbf{z}, t)\right|_{t=1}=p_{\boldsymbol{\alpha}}(\mathbf{z})
\end{array}\right.
$$

and

$$
\left\{\begin{array}{l}
\frac{\partial p_{1}(\mathbf{z}, t)}{\partial t}+\sum_{j=1}^{m} \frac{\partial}{\partial z_{j}}\left\{E\left[H_{j} \mid \mathbf{z}(t)=z\right] p_{0}(\mathbf{z}, t)\right. \\
\left.+E\left[D_{j} \mid \mathbf{z}(t)=\mathbf{z}\right] p_{1}(\mathbf{z}, t)\right\}=0 \\
\left.p_{1}(\mathbf{z}, t)\right|_{t=1}=0
\end{array}\right.
$$

to obtain $p\left(\mathbf{z}, t_{0}\right)=p_{0}\left(\mathbf{z}, t_{0}\right)+\varepsilon p_{1}\left(\mathbf{z}, t_{0}\right)$.

(e) Use (1-7) to obtain the density function $p_{\mathbf{x}\left(t_{0}\right)}(\mathbf{x})$.

When $\alpha(\omega), \xi_{1}(t, \omega)$ are independent, (4-12) reduces to the simple form $E\left[\boldsymbol{\xi}_{1}(t, \omega) \mid \boldsymbol{\alpha}(\omega)=\mathbf{q}(\mathbf{z}, t)\right]=E\left[\boldsymbol{\xi}_{1}(t, \omega)\right]$. It is often the case that $E\left[\boldsymbol{\xi}_{1}\right]=0$; then from (4-11) we know $E\left[H_{j} \mid \mathbf{z}(t)=\mathbf{z}\right]=O(\varepsilon)$, and from (4-15) we have $p_{1}(\mathbf{z}, t)=0$. Then the preceding analysis produces only the leading term $p_{0}(\mathbf{z}, t)$. In order to obtain any improvement, it is necessary to keep the terms up to order $\varepsilon^{2}$.

Instead of (4-6), we now have

$$
z_{j}(t)-z_{j}(t-\Delta t)=\left(D_{j}+\varepsilon H_{j}+\varepsilon^{2} W_{j}\right) \Delta t+O\left(\Delta t^{2}\right)+O\left(\varepsilon^{3}\right),
$$


where $D_{j}, H_{j}$ can still be expressed by (4-7), (4-8), but

$$
\begin{aligned}
& W_{j}=\left[\frac{-t_{0}}{1-t_{0}} \sum_{i, k=1}^{m} a_{j i} f_{i x_{k}}^{\prime}\left(T, \mathbf{x}_{0}(T), \boldsymbol{\xi}_{0}(T)\right) x_{2 k}(T)\right. \\
& \left.+\sum_{i, k=1}^{m} b_{j i} f_{i x_{k}}^{\prime}\left(t, \mathbf{x}_{0}(t), \boldsymbol{\xi}_{0}(t)\right) x_{2 k}(t)\right] \\
& +\left[\frac{-t_{0}}{1-t_{0}} \sum_{i=1}^{m} \sum_{k=1}^{n} a_{j i} f_{i \xi_{k}}^{\prime}\left(T, \mathbf{x}_{0}(T), \xi_{0}(T)\right) \xi_{2 k}(T, \omega)\right. \\
& \left.+\sum_{i=1}^{m} \sum_{k=1}^{n} b_{j k} f_{i \xi_{k}}^{\prime}\left(t, \mathbf{x}_{0}(t), \boldsymbol{\xi}_{0}(t)\right) \xi_{2 k}(t, \omega)\right] \\
& +\frac{1}{2}\left[\frac{-t_{0}}{1-t_{0}} \sum_{i=1}^{m} \sum_{l, k=1}^{n} a_{j i} f_{i \xi_{\zeta} \xi_{k}}^{\prime \prime}\left(T, \mathbf{x}_{0}(t), \boldsymbol{\xi}_{0}(T)\right) \xi_{1 l}(T, \omega) \xi_{1 k}(T)\right] \\
& \left.+\sum_{i=1}^{m} \sum_{l, k=1}^{n} b_{j i} f_{i \xi_{l} \xi_{k}}^{\prime \prime}\left(t, \mathbf{x}_{0}(t), \xi_{0}(t)\right) \xi_{1 l}(t, \omega) \xi_{1 k}(t, \omega)\right] \\
& +\frac{1}{2}\left[\frac{-t_{0}}{1-t_{0}} \sum_{i, l, k=1}^{m} a_{j i} f_{i x_{l} x_{k}}^{\prime \prime}\left(T, \mathbf{x}_{0}(T), \xi_{0}(T)\right) x_{1 l}(T) x_{1 k}(T)\right. \\
& \left.+\sum_{i, l, k=1}^{m} b_{j i} f_{i x_{l} x_{k}}^{\prime \prime}\left(t, \mathbf{x}_{0}(t), \boldsymbol{\xi}_{0}(t)\right) x_{1 l}(t) x_{1 k}(t)\right] \\
& +\left[\frac{-t_{0}}{1-t_{0}} \sum_{i, l=1}^{m} \sum_{k=1}^{n} a_{j i} f_{i x_{1} \xi_{k}}^{\prime \prime}\left(T, \mathbf{x}_{0}(T), \xi_{0}(T)\right) x_{1 l}(T) \xi_{1 k}(T, \omega)\right. \\
& \left.+\sum_{i, l=1}^{m} \sum_{k=1}^{n} b_{j i} f_{i x_{l} \xi_{k}}^{\prime \prime}\left(t, \mathbf{x}_{0}(t), \boldsymbol{\xi}_{0}(t)\right) x_{1 l}(t) \xi_{1 k}(t, \omega)\right],
\end{aligned}
$$

where $a_{j i}, b_{j i}, f_{i}, x_{1 k}, x_{2 k}, \xi_{1 k}, \xi_{2 k}$ are the components of $\mathbf{A}, \mathbf{B}, \mathbf{f}, \mathbf{x}_{1}, \mathbf{x}_{2}, \boldsymbol{\xi}_{1}, \boldsymbol{\xi}_{2}$, respectively.

Using an argument similar to the one above, we find that, under some boundedness assumptions, the equation $(1-8)$ can again be reduced to the first-order equation (4-13), but

$$
\begin{aligned}
\alpha_{j}(\mathbf{z}, t) & =\lim _{\Delta t \rightarrow 0} \frac{1}{\Delta t} E\left\{\left[z_{j}(t)-z_{j}(t-\Delta t)\right] \mid \mathbf{z}(t)=\mathbf{z}\right\} \\
& =E\left[D_{j} \mid \mathbf{z}(t)=\mathbf{z}\right]+\varepsilon E\left[H_{j} \mid \mathbf{z}(t)=\mathbf{z}\right]+\varepsilon^{2} E\left[W_{j} \mid \mathbf{z}(t)=\mathbf{z}\right],
\end{aligned}
$$

where terms with order greater than two have been omitted. As before,

$$
E\left[D_{j} \mid \mathbf{z}(t)=\mathbf{z}\right]=\left.D_{j}\right|_{\substack{\mathbf{x}_{0}(T)=\mathbf{x}_{0}(T, \mathbf{z}, t) \\ \mathbf{x}_{0}(t)=\mathbf{x}_{0}(t, \mathbf{z}, t)}},
$$


and if we notice that there is a coefficient $\varepsilon^{2}$ before $E\left[W_{j} \mid \mathbf{z}(t)=\mathbf{z}\right]$, then it can also be evaluated in a simple way, in which all the terms $E\left[\xi_{1 s} \xi_{1 k} \mid \mathbf{z}(t)=\mathbf{z}\right]$ are reduced to $E\left[\xi_{1 s} \xi_{1 k} \mid \boldsymbol{\alpha}(\omega)=\mathbf{q}(\mathbf{z}, t)\right]$. The term $E\left[H_{j} \mid \mathbf{z}(t)=\mathbf{z}\right]$ can be evaluated from (4-8) and (4-3), if we can find an efficient way to obtain $E\left[\xi_{1}(\tau, \omega) \mid \mathbf{z}(t)=\mathbf{z}\right]$ up to order $\varepsilon$.

By an argument similar to that used for the initial value problem [11], we find that the procedure for evaluating $E\left[\xi_{1 s}(\tau, \omega) \mid \mathbf{z}(t)=\mathbf{z}\right]$ up to order $\varepsilon$ is as follows:

(a) For the problem

$$
\left\{\begin{array}{l}
\frac{d \mathbf{x}(t)}{d t}=\mathbf{f}\left(t, \mathbf{x}(t), \boldsymbol{\xi}_{0}(t)\right)+\varepsilon \mathbf{f}_{\xi}\left(t, \mathbf{x}(t), \boldsymbol{\xi}_{0}(t)\right) \boldsymbol{\xi}_{1}(t, \omega) \\
\mathbf{A x}(0)+\mathbf{B x}(1)=\boldsymbol{\alpha}(\omega)
\end{array}\right.
$$

use the procedure discussed above to obtain the density function of $\mathbf{z}(t)$ up to order $\varepsilon$, and let it be $p_{\mathbf{z}(t)}=p_{0}(\mathbf{z}, t)+\varepsilon p_{1}(\mathbf{z}, t)$.

(b) For the auxiliary problem

$$
\left\{\begin{array}{l}
\frac{d \mathbf{x}^{*}(t, \tau)}{d t}=\mathbf{f}\left(t, \mathbf{x}^{*}(t, \tau), \boldsymbol{\xi}_{0}(t)\right)+\varepsilon \mathbf{f}_{\xi}\left(t, \mathbf{x}^{*}(t, \tau), \boldsymbol{\xi}_{0}(t)\right) \boldsymbol{\xi}_{1}(t, \omega) \xi_{1 s}(\tau, \omega), \\
\mathbf{A x}^{*}(0, \tau)+\mathbf{B x}^{*}(1, \tau)=\boldsymbol{\alpha}(\omega)
\end{array}\right.
$$

again use the procedure discussed above to obtain the density function of $\mathbf{z}^{*}(t, \tau)$ up to order $\varepsilon$, and let it be

$$
p_{\mathbf{z}^{*}(t, \tau)}=p_{0}^{*}(\mathbf{z}, t, \tau)+\varepsilon p_{1}^{*}(\mathbf{z}, t, \tau)
$$

where

$$
\mathbf{z}^{*}(t, \tau)=\mathbf{A} \mathbf{X}^{*}(T, \tau)+\mathbf{B} \mathbf{x}^{*}(t, \tau)
$$

(c) Use the following formula to evaluate

$$
\begin{aligned}
& E\left[\xi_{1 s}(\tau, \omega) \mid \mathbf{z}(t)=\mathbf{z}\right] \\
& \quad=E\left[\xi_{1 s}(\tau, \omega)\right]+\varepsilon \frac{p_{1}^{*}(\mathbf{z}, t, \tau)-p_{1}(\mathbf{z}, t) E\left[\xi_{1 s}(\tau, \omega)\right]}{p_{0}(\mathbf{z}, t)} .
\end{aligned}
$$

When $E\left[D_{j} \mid \mathbf{z}(t)=\mathbf{z}\right], E\left[H_{j} \mid \mathbf{z}(t)=\mathbf{z}\right]$, and $E\left[W_{j} \mid \mathbf{z}(t)=\mathbf{z}\right]$ have been evaluated, from (4-17) we can obtain the coefficients $\alpha_{j}$, and then we can find the density function of $\mathbf{x}(t)$ up to order $\varepsilon^{2}$ by a procedure similar to that discussed above.

5. Example. We consider the simple example

$$
\begin{gathered}
\left\{\begin{array}{l}
\dot{x}=1+\xi(t, \omega), \\
\dot{y}=x,
\end{array}\right. \\
\left\{\begin{array}{l}
x(0)=\alpha(\omega), \\
y(1)=\beta(\omega),
\end{array}\right.
\end{gathered}
$$

where $\xi(t, \omega)=\varepsilon \xi_{1}(t, \omega), E\left[\xi_{1}(t, \omega)\right]=t, E\left[\xi_{1}\left(t_{1}, \omega\right) \xi_{1}\left(t_{2}, \omega\right)\right]=\exp \left[-\left|t_{1}-t_{2}\right|\right]$, and $(\alpha(\omega), \beta(\omega))^{\mathrm{T}}$ is assumed to be independent of $\xi_{1}(t, \omega)$. This example was chosen so that the procedure outlined in this paper can be carried out analytically.

If we are interested in the case that $t_{0}=0$, then the auxiliary vector is $\mathbf{z}(t)=$ $[x(0), y(t)]^{\mathrm{T}}$ and the first-order partial differential equation (4-13) becomes

$$
\frac{\partial p(\mathbf{z}, t)}{\partial t}+\frac{\partial}{\partial z_{2}}\left[\alpha_{2}(\mathbf{z}, t) \cdot p(\mathbf{z}, t)\right]=0
$$

where $\mathbf{z}=\left(z_{1}, z_{2}\right)^{\mathrm{T}}$. 
If we let $x(t)=x_{0}(t)+\varepsilon x_{1}(t)+\varepsilon^{2} x_{2}(t), y(t)=y_{0}(t)+\varepsilon y_{1}(t)+\varepsilon^{2} y_{2}(t)$, then from (4-17) and (5-1a) we have

$$
\begin{aligned}
\alpha_{2}(\mathbf{z}, t) & =E[\dot{y}(t) \mid \mathbf{z}(t)=\mathbf{z}]=E[x(t) \mid \mathbf{z}(t)=\mathbf{z}] \\
& =E\left[x_{0}(t) \mid \mathbf{z}(t)=\mathbf{z}\right]+\varepsilon E\left[x_{1}(t) \mid \mathbf{z}(t)=\mathbf{z}\right]+\varepsilon^{2} E\left[x_{2}(t) \mid \mathbf{z}(t)=\mathbf{z}\right] .
\end{aligned}
$$

Under the condition $\mathbf{z}(t)=\mathbf{z}$ we have

or

$$
\left\{\begin{array}{l}
\frac{d\left(x_{0}+\varepsilon x_{1}+\varepsilon^{2} x_{2}\right)}{d t}=1+\varepsilon \xi_{1}(t, \omega), \\
\frac{d\left(y_{0}+\varepsilon y_{1}+\varepsilon^{2} y_{2}\right)}{d t}=\left(x_{0}+\varepsilon x_{1}+\varepsilon^{2} x_{2}\right),
\end{array}\right.
$$

$$
\begin{gathered}
\left\{\begin{array} { l } 
{ \frac { d x _ { 0 } } { d t ^ { * } } = 1 , } \\
{ \frac { d y _ { 0 } } { d t ^ { * } } = x _ { 0 } , }
\end{array} \quad \left\{\begin{array}{l}
x_{0}(0)=z_{1}, \\
y_{0}(t)=z_{2} ;
\end{array}\right.\right. \\
\left\{\begin{array} { l } 
{ \frac { d x _ { 1 } } { d t ^ { * } } = \xi _ { 1 } ( t ^ { * } , \omega ) , } \\
{ \frac { d y _ { 1 } } { d t ^ { * } } = x _ { 1 } , }
\end{array} \quad \left\{\begin{array}{l}
x_{1}(0)=0, \\
y_{1}(t)=0 ;
\end{array}\right.\right.
\end{gathered}
$$

and

$$
\left\{\begin{array} { l } 
{ \frac { d x _ { 2 } } { d t ^ { * } } = 0 , } \\
{ \frac { d y _ { 2 } } { d t ^ { * } } = x _ { 2 } , }
\end{array} \quad \left\{\begin{array}{l}
x_{2}(0)=0 \\
y_{2}(t)=0
\end{array}\right.\right.
$$

where the variable $t^{*}$ has been used to avoid confusion.

From $(5-4 \mathrm{c})$ we easily find that $x_{2}\left(t^{*}, \mathbf{z}, t\right)=0, y_{2}\left(t^{*}, \mathbf{z}, t\right)=0$, and from $(5-4 \mathrm{a})$, $(5-4 b)$ we have

and

$$
\left\{\begin{array}{l}
x_{0}\left(t^{*}, \mathbf{z}, t\right)=t^{*}+z_{1} \\
y_{0}\left(t^{*}, \mathbf{z}, t\right)=\frac{t^{* 2}-t^{2}}{2}+z_{1}\left(t^{*}-t\right)+z_{2},
\end{array}\right.
$$

$$
\left\{\begin{array}{l}
x_{1}\left(t^{*}, \mathbf{z}, t\right)=\int_{0}^{t^{*}} \xi_{1}(\tau, \omega) d \tau, \\
y_{1}\left(t^{*}, \mathbf{z}, t\right)=-\int_{t^{*}}^{t} d \tau \int_{0}^{\tau} \xi_{1}\left(\tau_{1}, \omega\right) d \tau_{1} .
\end{array}\right.
$$

If we substitute these results into (5-3), we have

$$
\alpha_{2}(\mathbf{z}, t)=\left[t+z_{1}\right]+\varepsilon \int_{0}^{t} E\left[\xi_{1}(\tau, \omega) \mid \mathbf{z}(t)=\mathbf{z}\right] d \tau .
$$

In order to evaluate $E\left[\xi_{1}(\tau, \omega) \mid \mathbf{z}(t)=\mathbf{z}\right]$ up to order $\varepsilon$ we use the procedure discussed in Sec. 4. First we consider the equation corresponding to (4-18):

$$
\begin{gathered}
\left\{\begin{array}{l}
\frac{d x}{d t}=1+\varepsilon \xi_{1}(t, \omega), \\
\frac{d y}{d t}=x,
\end{array}\right. \\
x(0)=\alpha(\omega), \quad y(1)=\beta(\omega) .
\end{gathered}
$$

According to the steps discussed in Sec. 4, we know that

$$
\psi\left(t^{*}\right)=\left(\begin{array}{cc}
1 & 0 \\
t^{*}-t & 1
\end{array}\right)
$$

and that the equation in this case is

$$
\frac{\partial p(\mathbf{z}, t)}{\partial t}+\left[t+z_{1}+\varepsilon \frac{t^{2}}{2}\right] \cdot \frac{\partial}{\partial z_{2}} p(\mathbf{z}, t)=0 .
$$


If we let the density function of $\mathbf{z}(t)$ up to order $\varepsilon$ be $p_{\mathbf{z}(t)}=p_{0}(\mathbf{z}, t)+\varepsilon p_{1}(\mathbf{z}, t)$, then from (5-9) and condition (1-6) we have

$$
\left\{\begin{array}{l}
p_{0}(\mathbf{z}, t)=p_{\alpha \beta}\left(z_{1}, z_{2}+(1-t) z_{1}+\frac{1-t^{2}}{2}\right), \\
p_{1}(\mathbf{z}, t)=\frac{1-t^{3}}{6} \frac{\partial p_{\alpha \beta}}{\partial \beta}\left(z_{1}, z_{2}+(1-t) z_{1}+\frac{1-t^{2}}{2}\right),
\end{array}\right.
$$

where $p_{\alpha \beta}(\alpha, \beta)$ is the known density function of $\alpha(\omega), \beta(\omega)$.

In order to consider the second-order correction term, we solve the new auxiliary equation corresponding to (4-19):

$$
\left\{\begin{array}{l}
\frac{d x^{*}(t, \tau)}{d t}=1+\varepsilon \xi_{1}(t, \omega) \xi_{1}(\tau, \omega) \\
\frac{d y^{*}(t, \tau)}{d t}=x^{*}(t, \tau)
\end{array}\right.
$$

with the condition $x^{*}(0, \tau)=\alpha(\omega), y^{*}(1, \tau)=\beta(\omega)$. If we denote the density function of $\mathbf{z}^{*}(t, \tau)=\left(x^{*}(0, \tau), y(t, \tau)\right)^{\mathrm{T}}$ by $p^{*}(\mathbf{z}, t, \tau)=p_{0}^{*}(\mathbf{z}, t, \tau)+\varepsilon p_{1}^{*}(\mathbf{z}, t, \tau)$, then in a similar way we have the first-order partial differential equation

$$
\frac{\partial p^{*}(\mathbf{z}, t, \tau)}{\partial t}+\left[t+z_{1}+\varepsilon \int_{0}^{t} e^{-\left|\tau_{1}-\tau\right|} d \tau_{1}\right] \cdot \frac{\partial}{\partial z_{2}} p^{*}(\mathbf{z}, t, \tau)=0
$$

and its solution

$$
\left\{\begin{array}{l}
p_{0}^{*}(\mathbf{z}, t, \tau)=p_{\alpha \beta}\left(z_{1}, z_{2}+(1-t) z_{1}+\frac{1-t^{2}}{2}\right), \\
p_{1}^{*}(\mathbf{z}, t, \tau)=\int_{t}^{1} \int_{0}^{\tau_{1}} e^{-\left|\tau_{2}-\tau\right|} d \tau_{2} d \tau_{1} \cdot \frac{\partial p_{u \beta}}{\partial \beta}\left(z_{1}, z_{2}+(1-t) z_{1}+\frac{1-t^{2}}{2}\right) .
\end{array}\right.
$$

From (4-20) we obtain

$$
\begin{aligned}
E\left\{\xi_{1}(\tau, \omega) \mid \mathbf{z}(t)=\mathbf{z}\right\}= & \tau+\varepsilon \cdot\left[\int_{t}^{1} \int_{0}^{\tau_{1}} e^{-\left|\tau_{2}-\tau\right|} d \tau_{2} d \tau_{1}-\tau \cdot \frac{1-t^{3}}{6}\right] \\
& \cdot \frac{\left(\partial p_{\alpha \beta} / \partial \beta\right)\left(z_{1}, z_{2}+(1-t) z_{1}+\left(1-t^{2}\right) / 2\right)}{p_{\alpha \beta}\left(z_{1}, z_{2}+(1-t) z_{1}+\left(1-t^{2}\right) / 2\right)} .
\end{aligned}
$$

Then from (5-6) we find that

$$
\begin{aligned}
\alpha_{2}(\mathbf{z}, t)= & {\left[t+z_{1}\right]+\varepsilon \int_{0}^{t} \tau d \tau+\varepsilon^{2} \cdot \frac{\left(\partial p_{\alpha \beta} / \partial \beta\right)\left(z_{1}, z_{2}+(1-t) z_{1}+\left(1-t^{2}\right) / 2\right)}{p_{\alpha \beta}\left(z_{1}, z_{2}+(1-t) z_{1}+\left(1-t^{2}\right) / 2\right)} } \\
& \cdot\left[\int_{0}^{t} d \tau \int_{t}^{1} d \tau_{1} \int_{0}^{\tau_{1}} e^{-\left|\tau_{2}-\tau\right|} d \tau_{2}-\frac{1-t^{3}}{6} \int_{0}^{t} \tau d \tau\right] \\
= & {\left[t+z_{1}\right]+\varepsilon \cdot \frac{t^{2}}{2}+\varepsilon^{2} \cdot \frac{\left(\partial p_{\alpha \beta} / \partial \beta\right)\left(z_{1}, z_{2}+(1-t) z_{1}+\left(1-t^{2}\right) / 2\right)}{p_{\alpha \beta}\left(z_{1}, z_{2}+(1-t) z_{1}+\left(1-t^{2}\right) / 2\right)} } \\
& \cdot\left[\int_{0}^{t} d \tau \int_{t}^{1} d \tau_{1} \int_{0}^{\tau_{1}} e^{-\left|\tau_{2}-\tau\right|} d \tau_{2}-\frac{t^{2}\left(1-t^{3}\right)}{12}\right] .
\end{aligned}
$$

If we substitute this into Eq. (5-2) and solve it under the condition (1-6) again, we obtain the solution, up to order $\varepsilon^{2}$,

$$
p_{\mathbf{z}(t)}(\mathbf{z}, t)=p_{0}(\mathbf{z}, t)+\varepsilon p_{1}(\mathbf{z}, t)+\varepsilon^{2} p_{2}(\mathbf{z}, t),
$$

where $p_{0}, p_{1}$ are as (5-10) and

$$
\begin{aligned}
p_{2}(\mathbf{z}, t)= & \frac{1}{2} \frac{\partial^{2} p_{\alpha \beta}}{\partial \beta^{2}}\left(z_{1}, z_{2}+(1-t) z_{1}+\frac{1-t^{2}}{2}\right) \\
& \cdot \int_{t}^{1} d \tau_{2} \int_{t}^{1} d \tau_{1} \int_{0}^{\tau_{1}} d \gamma_{2} \int_{0}^{\tau_{2}} e^{-\left|\gamma_{2}-\gamma_{1}\right|} d \gamma_{1} .
\end{aligned}
$$


At last from (1-7) we obtain the density function of $x(0), y(0)$

$$
p(x, y)=\left.\left\{p_{0}(\mathbf{z}, 0)+\varepsilon p_{1}(\mathbf{z}, 0)+\varepsilon^{2} p_{2}(\mathbf{z}, 0)\right\}\right|_{\mathbf{z}=(x, y)^{\mathrm{T}}}
$$

up to order $\varepsilon^{2}$.

Acknowledgment. The author dedicates this paper to the late Professor Richard C. DiPrima, as a sign of his respect and in recognition of many kindnesses shown the author while he was at Rensselaer.

\section{REFERENCES}

[1] L. Arnold, Stochastic differential equations: Theory and applications, John Wiley \& Sons, New York (1974)

[2] W. E. Boyce and Ning-Mao Xia, The approach to normality of the solutions of random boundary and eigenvalue problems with weakly correlated coefficients, Quart. Appl. Math. 40, 419-445 (1983)

[3] W. H. Fleming, Stochastic control for small noise intensities, SIAM J. Control 9, 473-517 (1971)

[4] I. I. Gihman and A. V. Skorohod, Stochastic differential equations, Springer-Verlag, New York (1972)

[5] R. Z. Khazminskii, On stochastic processes defined by differential equations with a small parameter, Theory Probab. Appl. 11, 211-228 (1966)

[6] M. D. Lax, The method of moments for linear random boundary value problems, SIAM J. Appl. Math. 31, 62-83 (1976)

[7] R. F. Pawula, Generalizations and extensions of the Fokker-Planck-Kolmogorov equations, IEEE Trans. Inform. Theory, IT-13, 33-41 (1967)

[8] T. T. Soong, Random differential equations in science and engineering, Academic Press, New York (1973)

[9] B. S. White and J. N. Franklin, A limit theorem for stochastic two-point boundary-value problems of ordinary differential equations, Comm. Pure Appl. Math. 32, 253-275 (1979)

[10] Ning-Mao Xia, W. E. Boyce, and M. R. Barry, Two-point boundary value problems containing a finite number of random variables, Stochastic Anal. Appl. 1, 117-137 (1983)

[11] Ning-Mao Xia and W. E. Boyce, The density function of the solution of a random initial value problem containing small stochastic processes, SIAM J. Appl. Math. 44, 1192-1209 (1984)

[12] Ning-Mao Xia, The solutions for the two-point boundary value problems of stochastic differential equations containing small white noises, Acta Math. Appl. 8, 340-350 (1985) 